\title{
SCIENTIFIC REPORTS

\section{OPEN Ultrathin perpendicular magnetic anisotropy CoFeB free layers for highly efficient, high speed writing in spin-transfer-torque magnetic random access memory}

Jodi M. Iwata-Harms*, Guenole Jan, Santiago Serrano-Guisan, Luc Thomas, Huanlong Liu, Jian Zhu, Yuan-Jen Lee, Son Le, Ru-Ying Tong, Sahil Patel, Vignesh Sundar, Dongna Shen, YiYang, Renren He, Jesmin Haq, Zhongjian Teng, Vinh Lam, Paul Liu, Yu-Jen Wang, Tom Zhong, Hideaki Fukuzawa \& Po-Kang Wang

Perpendicular magnetic anisotropy (PMA) ferromagnetic CoFeB with dual MgO interfaces is an attractive material system for realizing magnetic memory applications that require highly efficient, high speed current-induced magnetic switching. Using this structure, a sub-nanometer CoFeB layer has the potential to simultaneously exhibit efficient, high speed switching in accordance with the conservation of spin angular momentum, and high thermal stability owing to the enhanced interfacial PMA that arises from the two CoFeB-MgO interfaces. However, the difficulty in attaining PMA in ultrathin CoFeB layers has imposed the use of thicker CoFeB layers which are incompatible with high speed requirements. In this work, we succeeded in depositing a functional CoFeB layer as thin as five monolayers between two MgO interfaces using magnetron sputtering. Remarkably, the insertion of $\mathrm{Mg}$ within the $\mathrm{CoFeB}$ gave rise to an ultrathin $\mathrm{CoFeB}$ layer with large anisotropy, high saturation magnetization, and good annealing stability to temperatures upwards of $400^{\circ} \mathrm{C}$. When combined with a low resistance-area product $\mathrm{MgO}$ tunnel barrier, ultrathin CoFeB magnetic tunnel junctions (MTJs) demonstrate switching voltages below $500 \mathrm{mV}$ at speeds as fast as $1 \mathrm{~ns}$ in $30 \mathrm{~nm}$ devices, thus opening a new realm of high speed and highly efficient nonvolatile memory applications.

PMA-MTJs composed of a $\mathrm{CoFeB}$ free layer and $\mathrm{MgO}$ tunnel barrier have yielded numerous breakthroughs including scalability, endurance, and high thermal stability ${ }^{1-9}$. From a technological perspective, CoFeB-MgO-based spin-transfer-torque magnetic random access memory (STT-MRAM) has emerged as the most promising alternative to existing silicon-based memory technologies. However, one prominent shortcoming of conventional CoFeB-MgO structures is the requirement for lower switching voltage at faster switching speeds. To attain a low switching voltage, the conservation of spin angular momentum stipulates the need for low magnetic moment which is embodied by an ultrathin ferromagnetic free layer. PMA in ultrathin Fe has been demonstrated $^{10}$, but the inability to achieve PMA in ultrathin $\mathrm{CoFeB}$ with dual $\mathrm{MgO}$ interfaces stems from the delicate balance between achieving layer continuity, preserving free layer metallicity, and retaining a low resistance-area product (RA) for the $\mathrm{MgO}$ layers. Here, we report the use of an Mg insertion layer within an ultrathin CoFeB layer that gives rise to PMA. The device performance of this ultrathin free layer was recently reported as it achieved breakthroughs in low magnetic moment and low damping that gave rise to ultra-low voltage and ultra-low power devices $^{11,12}$. In this work, we show that the achievement of ultrathin CoFeB layers are of technological importance as they fulfill the requirements for reversal of the free layer magnetization at short pulse lengths below $10 \mathrm{~ns}$, and at deep error rates of less than $1 \mathrm{ppm}$ which are essential to applications such as last level cache that use static random access memory technology. 
(a)

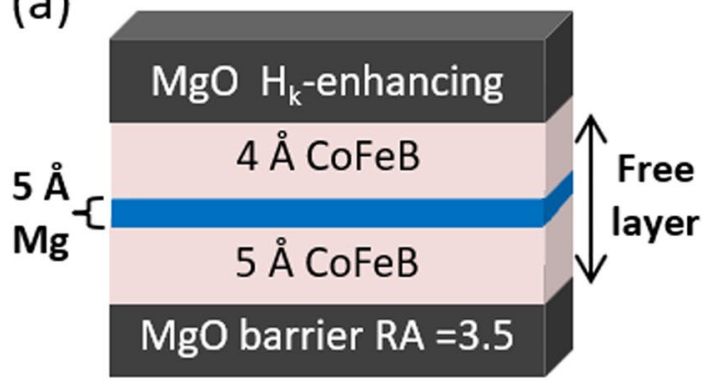

(b)

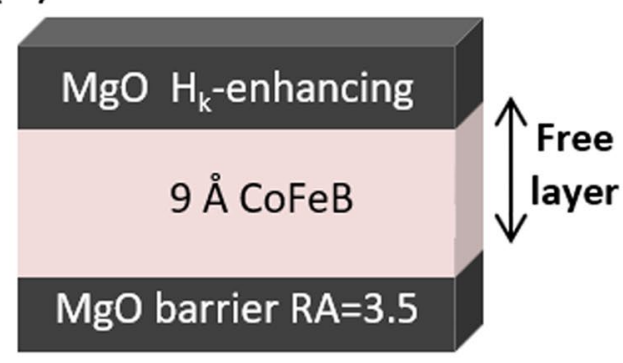

Figure 1. Schematics of ultrathin $9 \AA$ CFB free layers. (a) A $9 \AA$ CFB free layer with a $5 \AA \mathrm{Mg}$ insertion layer. Layer thicknesses are not drawn to scale. (b) A $9 \AA \AA$ CFB free layer without an Mg insertion. Layer thicknesses are not drawn to scale. The MgO barrier of the MTJ stacks was engineered for a low RA of $3.5 \Omega \cdot \mu \mathrm{m}^{2}$.
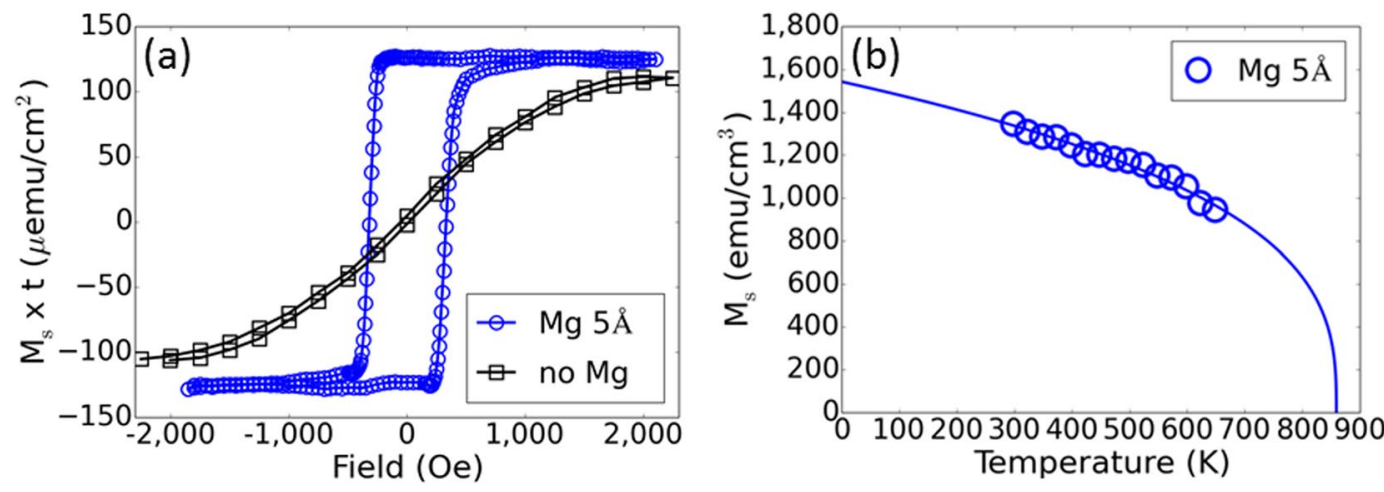

Figure 2. Magnetic properties of ultrathin $9 \AA$ CFB free layers. (a) Magnetic moment as a function of applied field for a $9 \AA$ CFB free layer with and without a $5 \AA \mathrm{Mg}$ insertion layer. Measurements were performed at room temperature. (b) Temperature dependence of $M_{s}$ for a $9 \AA$ CFB free layer with $5 \AA$ Mg insertion layer.

Conventional free layers use thicker $\mathrm{CoFeB}$ (CFB) layers that consist of refractory metal interfaces or insertions ${ }^{13-15}$. These free layers can exhibit good thermal stability withstanding $400^{\circ} \mathrm{C}$ back end of line process temperatures and a wide range of operating temperatures, but require a high switching voltage. By contrast, the conservation of spin angular momentum argues that a lower moment free layer is important for the design of low switching voltage magnetic random access memory (MRAM) devices ${ }^{12,16}$. This study shows that ultrathin, thermally stable, high saturation magnetization CFB free layers can be achieved with the incorporation of $\mathrm{Mg}$, enabling a low switching voltage at short pulse lengths. The effect of $\mathrm{Mg}$ is remarkable as PMA is observed in CFB free layers with thicknesses $t_{F L}$ as low as $6.6 \AA$, whereas in the absence of Mg, PMA is first observed for an $11 \AA ̊$ CFB free layer. For a $30 \mathrm{~nm}$ device, estimates of the upper limit of the energy barrier $E_{b}$ between parallel and anti-parallel states are large, thus good thermal stability is expected up to the maximum operating temperature of $85^{\circ} \mathrm{C}$. Experimentally, nominal $30 \mathrm{~nm}$ devices with a $9 \AA$ free layer exhibit sharp switching of the free layer magnetization with respect to applied field or current, and a very low RA of $3.5 \Omega \cdot \mu \mathrm{m}^{2}$ with a tunnel magnetoresistance ratio (TMR) up to $150 \%$. High speed testing revealed these devices are capable of switching at $1 \mathrm{~ns}$ with a writing voltage below $500 \mathrm{mV}$, and below $300 \mathrm{mV}$ for pulse lengths ranging from 3 to $50 \mathrm{~ns}$. These properties highlight the promise of PMA-MTJs, consisting of ultrathin CFB free layers with dual $\mathrm{MgO}$ interfaces, for expanding MRAM into new memory applications.

\section{Results}

We report on full MTJ film stacks that include a seed layer, synthetic antiferromagnet reference layer, MgO barrier, $\mathrm{CFB}$ free layer, $\mathrm{MgO} \mathrm{H}_{k}$-enhancing layer, and nitride-based cap. As depicted in Fig. 1, two variations of the $\mathrm{CoFeB}$ free layer are investigated. Each free layer is an ultrathin, $9 \AA \mathrm{CoFeB}$ free layer that consists of Fe-rich $(\mathrm{CoFe})_{1-y} \mathrm{~B}_{y}$, where the Co:Fe ratio is $1: 2.7$ and $y$ is 24 percent. The first free layer variation, shown in Fig. 1a, depicts the insertion of an $\mathrm{Mg}$ layer with a nominal thickness of $5 \AA$ within the CFB free layer. The second variation, illustrated in Fig. 1b, omits the Mg layer in favor a continuous CFB layer. All samples are annealed at $400^{\circ} \mathrm{C}$ for 3.5 hours after the deposition of the MTJ stack was complete.

We use vibration sample magnetometry (VSM) to measure the magnetic properties of the free layer along the out-of-plane easy direction at temperatures between $25^{\circ} \mathrm{C}$ and $375^{\circ} \mathrm{C}$. Figure 2a displays hysteresis loops of magnetic moment versus applied magnetic field measured at room temperature on blanket films. In the absence of $\mathrm{Mg}$, a $9 \AA \mathrm{CoFeB}$ free layer does not exhibit PMA; whereas, the addition of $5 \AA$ of $\mathrm{Mg}$ clearly exhibits free layer PMA. Furthermore, the addition of $\mathrm{Mg}$ results in a slightly higher $M_{s} \times t_{F L}$ than a free layer without Mg consistent with better magnetic properties. Figure $2 \mathrm{~b}$ shows that the addition of $\mathrm{Mg}$ to $\mathrm{CFB}$ results in a room 

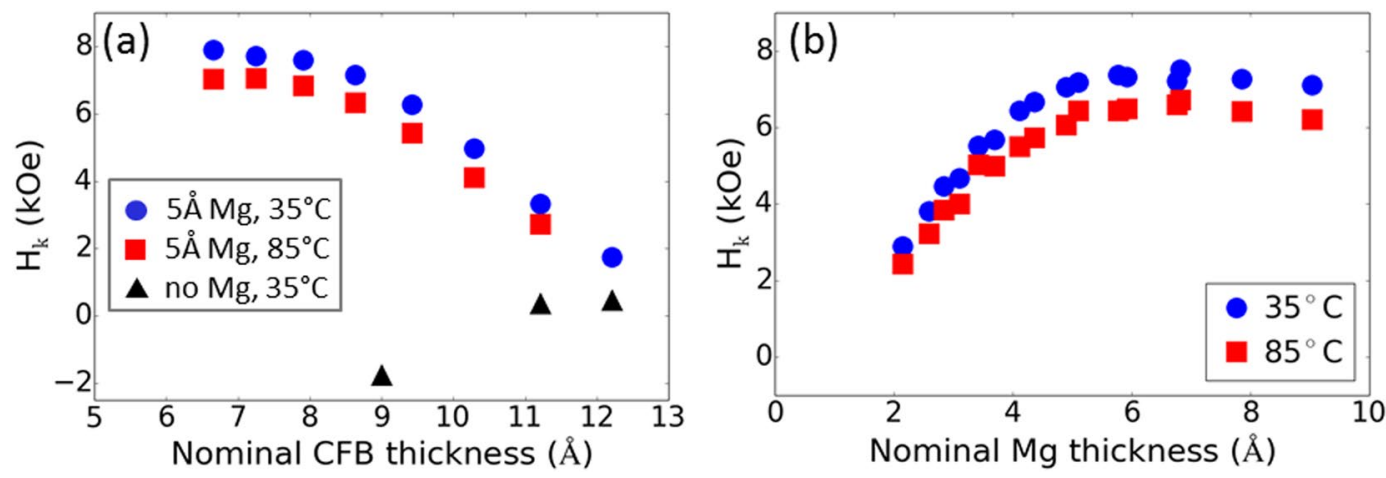

Figure 3. $H_{k}$ variation with free layer thickness and temperature. (a) $H_{k}$ dependence on nominal CFB thickness measured using FMR at $35^{\circ} \mathrm{C}$ and $85^{\circ} \mathrm{C}$, with and without an $\mathrm{Mg}$ insertion layer. (b) $\mathrm{H}_{k}$ dependence on nominal $\mathrm{Mg}$ thickness measured using FMR at $35^{\circ} \mathrm{C}$ and $85^{\circ} \mathrm{C}$.

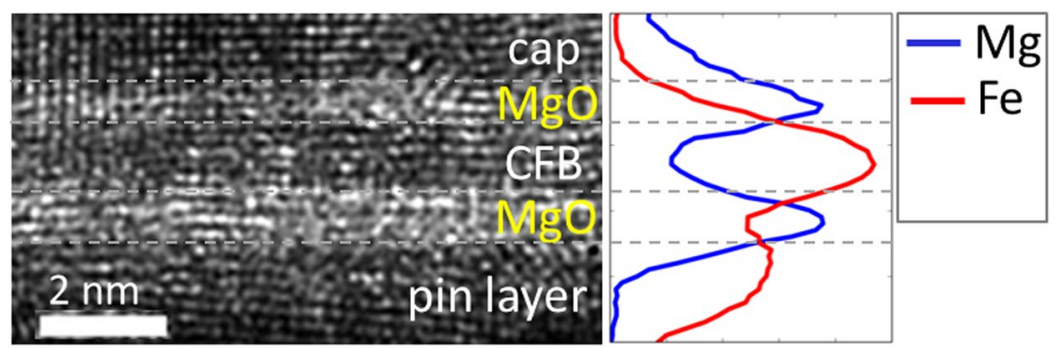

Figure 4. Cross-sectional TEM image of a $9 \AA$ AFB free layer with EDS line profile. TEM and EDS cannot confirm the presence of a distinct Mg layer within the CFB free layer. Dashed lines serve as a guide to the eye.

temperature saturation magnetization $M_{s}$ of $1350 \mathrm{emu} / \mathrm{cm}^{3}$ with $M_{s}$ disappearing at $860 \mathrm{~K}\left(T_{M_{s}}=0\right)$. The temperature dependence of the free layer $M_{s}$ is fitted by the $T^{1 / 3}$ power law indicated by the solid line, where $M_{s}(T)=M_{0} \times\left(1-\frac{T}{T_{M_{s}}=0}\right) \frac{1}{3}$, and is consistent with previous studies of CFB free layers and other ferromagnets ${ }^{15,17,18}$.

The anisotropy field $H_{k}$ measured by ferromagnetic resonance spectroscopy (FMR) as a function of the free layer $\mathrm{CFB}$ and $\mathrm{Mg}$ thickness are depicted with solid symbols in Fig. 3a,b, respectively. In Fig. 3a, free layer PMA and $H_{k}$ demonstrate a strong dependence on the thickness of CFB and the presence of $\mathrm{Mg}$. Figure $3 \mathrm{a}$ reveals a decrease in $H_{k}$ with CFB thickness due to increasing free layer magnetic moment, $M_{s} \times t_{F L}$. Interestingly, the addition of $\mathrm{Mg}$ to the free layer demonstrates PMA for free layer thicknesses as thin as $6.6 \AA$ and up to $12.2 \AA$, the lower and upper limits of this study, respectively. In contrast, Fig. 2a displayed the absence of PMA for a no-Mg $9 \AA$ CFB free layer. Without Mg, the triangular symbols in Fig. 3a assert that PMA occurs at an increased CFB thickness of $11 \AA$ with $H_{k}$ less than 500 Oe at $35^{\circ} \mathrm{C}$. Below this critical thickness, the CFB free layer exhibits in-plane anisotropy. These observations are important as they show that the addition of $\mathrm{Mg}$ extends the range of PMA in CFB free layers to ultrathin thickness levels required for low switching voltages in PMA-MTJs.

Next, Fig. $3 \mathrm{~b}$ demonstrates the sensitivity of the free layer $H_{k}$ on $\mathrm{Mg}$ content. Over the nominal $\mathrm{Mg}$ thickness range of this study, $2 \AA$ to $9 \AA$, a $9 \AA$ CFB free layer demonstrates PMA for all Mg thicknesses. $H_{k}$ sharply increases from a nominal Mg thickness of $2 \AA$ reaching a maximum near $7 \AA$. From $7 \AA$ to $9 \AA$, the $H_{k}$ dependence on $\mathrm{Mg}$ weakened suggesting an upper limit for $\mathrm{Mg}$. This upper limit, and more generally, the range of PMA enabled by the insertion of $\mathrm{Mg}$ can be tuned by the oxidation conditions of the adjacent $\mathrm{MgO}$ barrier and $\mathrm{H}_{k}$-enhancing layer, nitridation conditions of the nitride-based cap, and $\mathrm{Mg}$ content of the often under-oxidized $\mathrm{H}_{k}$-enhancing layer.

To better understand the role of the Mg insertion, Fig. 4 shows a cross-sectional transmission electron microscopy (TEM) micrograph and a corresponding energy dispersive spectroscopy (EDS) line scan of a $9 \AA$ CFB free layer with a $5 \AA \mathrm{Mg}$ insertion layer. The microstructure of the free layer depicted in the TEM micrograph reveals a continuous, crystalline layer with clear rows of atoms. The ultrathin free layer is believed to benefit from templating effects of the neighboring (001) $\mathrm{MgO}$ barrier and $H_{k}$-enhancing layers, such that the (001) texture appears to extend from the $\mathrm{MgO}$ barrier into the nitride-based cap. Surprisingly, both the TEM micrograph and EDS line scan could not confirm the presence of a distinct Mg insertion layer. Therefore, TEM and EDS were extended to an as-deposited sample to understand the effects of annealing on Mg diffusion. Again, unexpectedly, the TEM micrograph and EDS line scan revealed the absence of an $\mathrm{Mg}$ layer. Since annealing is not the reason for the absence of the Mg layer, we attribute the absence of the Mg layer to the low binding energy of the Mg-Mg bond (Table 1) that results in the loss of $\mathrm{Mg}$ through re-sputtering or the intermixing of $\mathrm{Mg}$ during the subsequent deposition of the $4 \AA \mathrm{CoFeB}$ layer. We also hypothesize that the $4 \AA$ of CFB may punch through the Mg layer 


\begin{tabular}{|l|l|}
\hline Bond & Bond Disassociation Energies $\left.^{\mathbf{2 2}} \mathbf{( k J / m o l}\right)$ \\
\hline $\mathrm{Mg}-\mathrm{Mg}$ & 11.3 \\
\hline $\mathrm{Fe}-\mathrm{Fe}$ & 118 \\
\hline $\mathrm{Co}-\mathrm{Co}$ & $<127$ \\
\hline
\end{tabular}

Table 1. Comparison of bond disassociation energies for constituent free layer atoms.

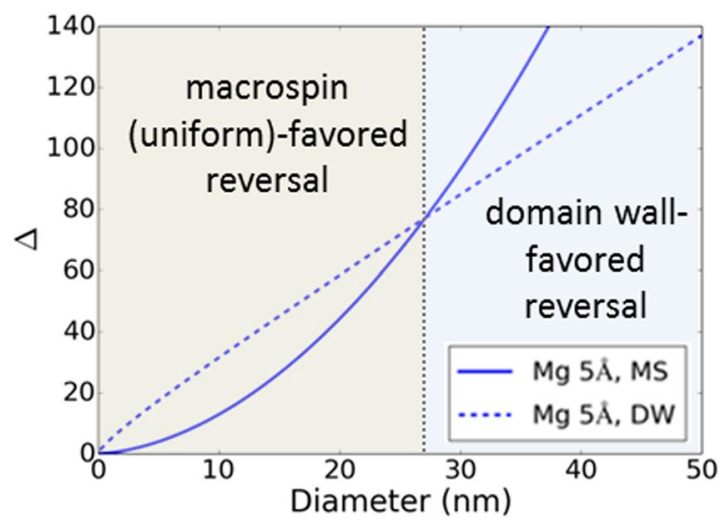

Figure 5. Simulated thermal stability factor $\Delta$ for different device diameters under macrospin and domain wall reversal models.

effectively implanting into the lower $5 \AA \mathrm{CoFeB}$ layer resulting in a "floating" Mg layer that is partially incorporated into the $\mathrm{MgO} \mathrm{H}_{k}$-enhancing layer. Although $\mathrm{Mg}$ cannot be accurately quantified in TEM and EDS, a small portion of $\mathrm{Mg}$ is believed to remain within the structure. This is supported by the free layer properties which exhibit a strong dependence on $\mathrm{Mg}$ content.

Our experimental results enable us to derive the effective anisotropy constant $K_{\text {eff }}$ and the interfacial anisotropy constant $K_{i}$ which are defined as: $K_{e f f}=\frac{M_{s} H_{k}}{2}=\frac{K_{i}}{t_{F L}}-2 \pi M_{s}^{2}$. At $25^{\circ} \mathrm{C}$, a $9 \AA$ CFB free layer with $\mathrm{Mg}$ insertion exhibits $M_{s}$ of $1350 \mathrm{emu} / \mathrm{cm}^{3}$ and $H_{k}$ of $7.3 \mathrm{kOe}$. These properties correspond to a free layer with $K_{\text {eff }} \times t_{F L}$ of $0.44 \mathrm{erg} / \mathrm{cm}^{2}$ and $K_{i}$ of $1.48 \mathrm{erg} / \mathrm{cm}^{2}$. The anisotropy constants of the $9 \AA$ CFB free layer with $\mathrm{Mg}$ are close to values previously reported for thicker free layers designed for high thermal stability and data retention over very wide operating temperature ranges ${ }^{15}$. Given the similarities of the anisotropy constants, MTJs consisting of a $9 \AA$ CFB free layer should exhibit good thermal stability represented by the thermal stability factor $\Delta$. Additionally, with a high $T_{M_{s}}=0$ and high $H_{k}$ at $85^{\circ} \mathrm{C}$ as shown in Fig. 3, MTJs consisting of a $9 \AA$ CFB free layer should also exhibit good data retention up to $85^{\circ} \mathrm{C}$.

Next, the results of these studies are discussed from the perspective of the requirements for PMA-MTJs for MRAM applications. We can approximate $\Delta$, defined as $\frac{E_{b}}{k_{B} T}$, using both magnetization reversal models for a circular $30 \mathrm{~nm}$ MTJ device fabricated with an ideal, damage-free fabrication process. Under the first model, switching can be described by the macrospin approximation (MS) in which the free layer magnetic moment rotates uniformly. In this case, the energy barrier is given by $E_{b, M S}=K_{e f f} S t_{F L}$, where $S$ is the device surface $\operatorname{area}^{19}$. For larger diameters, magnetization reversal is mediated by the nucleation and propagation of a domain wall (DW) across the device leading to the following expression for the energy barrier ${ }^{20}: E_{b, D W}=4 d t_{F L} \sqrt{A \times K_{e f f}}$, where $d$ is the device diameter and $A$ is the exchange stiffness constant. The exchange stiffness constant $A$ varies as $M_{s}^{2}$ such that $A=A_{0, \mathrm{CoFe}}\left(\frac{M_{s, F L}}{M_{0, \mathrm{CoFe}}}\right)^{2}$, where $A_{0, \mathrm{CoFe}}=35.8 \times 10^{-7} \mathrm{erg} / \mathrm{cm}$ and $M_{0, \mathrm{CoFe}}=1946 \mathrm{emu} / \mathrm{cm}^{315,21}$. In order to calculate $E_{b}$, we must account for the reduction of the demagnetization factor as it relates to $H_{k}$ using the expressions discussed in ref. ${ }^{15}$. This relationship finds that $H_{k}$ for a $30 \mathrm{~nm}$ diameter device is $8.9 \mathrm{kOe}$ at $25^{\circ} \mathrm{C}$, and $7.8 \mathrm{kOe}$ at $85^{\circ} \mathrm{C}$. Under a macrospin model of magnetic reversal, a $30 \mathrm{~nm}$ device exhibits a high $\Delta$ of 93 at $25^{\circ} \mathrm{C}$ that decreases to 65 at $85^{\circ} \mathrm{C}$. Under a domain wall model of magnetic reversal, a $30 \mathrm{~nm}$ device also exhibits a high $\Delta$ of 85 at $25^{\circ} \mathrm{C}$ that decreases to 61 at $85^{\circ} \mathrm{C}$. The estimated values of $\Delta$ indicate for a $30 \mathrm{~nm}$ device, magnetization reversal for a $9 \AA$ CFB free layer occurs by domain wall nucleation and propagation. The crossover for switching mechanisms occurs at a device diameter of $27 \mathrm{~nm}$ as shown in Fig. 5. In any case, both reversal models show that ultrathin CFB free layer MTJs are capable of high thermal stability for $30 \mathrm{~nm}$ devices between $25^{\circ} \mathrm{C}$ and $85^{\circ} \mathrm{C}$. However, as $\Delta$ assumes a perfect patterning process in which the cylindrical MTJ pillars incur no damage, the estimated values represent the upper limit for $\Delta$. A previously reported study finds $\Delta_{M S}=59$ at $25^{\circ} \mathrm{C}$ and the difference between the estimated $\Delta$ and experimental $\Delta$ is attributed to MTJ pillar damage incurred during fabrication ${ }^{12}$.

Next, we examine the switching characteristics of nominal $30 \mathrm{~nm}$ diameter ultrathin CFB free layer MTJ devices. Figure 6a depicts a resistance versus magnetic field (R-H) loop measured with an applied DC bias of $20 \mathrm{mV}$. The R-H loop exhibits sharp switching of the free layer between high and low resistance states 

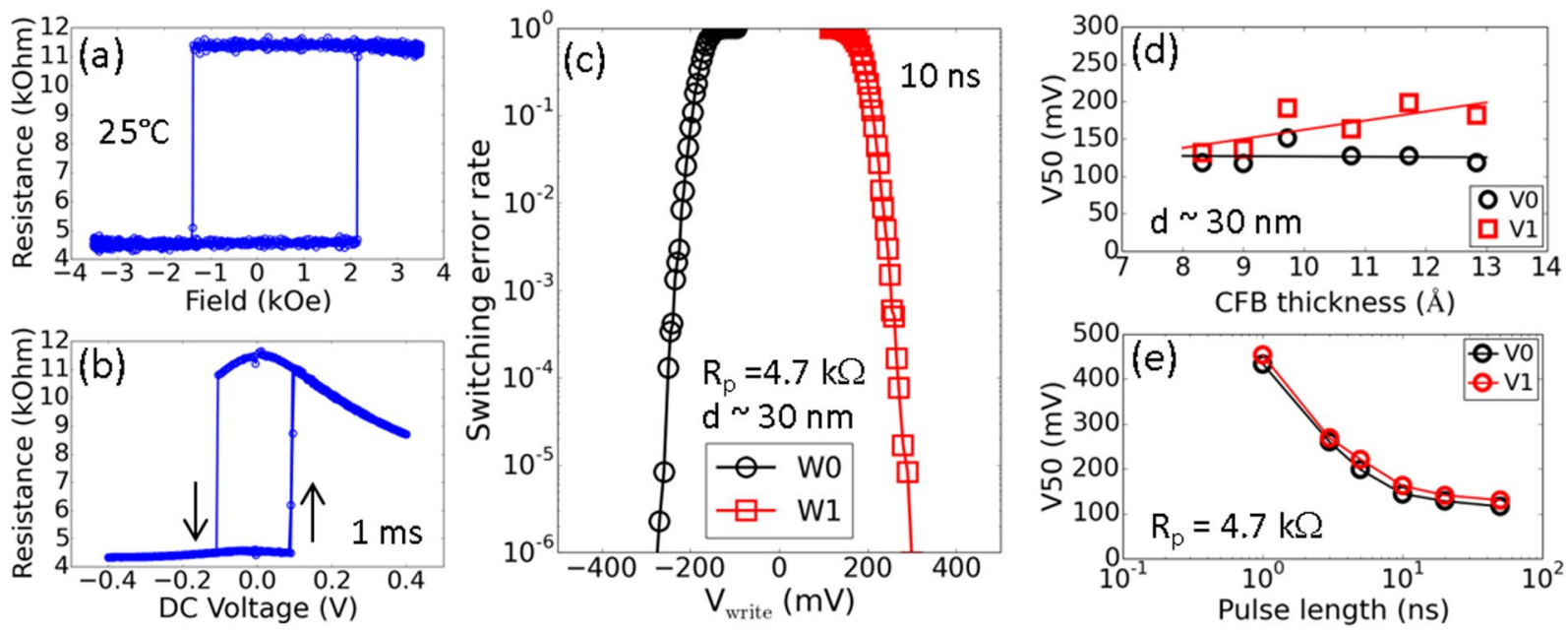

Figure 6. Room temperature device switching characteristics. (a) Resistance versus applied magnetic field and (b) resistance versus DC voltage (1 ms pulses) curves from a nominal $30 \mathrm{~nm}$ device. (c) Switching error rate of a nominal $30 \mathrm{~nm}$ device measured with $10 \mathrm{~ns}$ pulses down to $1 \mathrm{ppm}$ error level. $50 \%$ probability switching voltage dependence for nominal $30 \mathrm{~nm}$ diameter devices on (d) CFB free layer thickness and (e) pulse length.

\begin{tabular}{|l|l|l|}
\hline Characteristic & \multicolumn{2}{|l|}{ This Work } \\
\hline Writing pulse length & $10 \mathrm{~ns}$ & $1 \mathrm{~ns}$ \\
\hline Device diameter (electrical CD) & nominal $30 \mathrm{~nm}$ \\
\hline $50 \%$ switching voltage, $\mathrm{W} 1$ & $162 \mathrm{mV}$ & $453 \mathrm{mV}$ \\
\hline $50 \%$ switching current & $34 \mu \mathrm{A}$ & $96 \mu \mathrm{A}$ \\
\hline Switching energy & $0.056 \mathrm{pJ}$ & $0.044 \mathrm{pJ}$ \\
\hline Thermal budget & $400^{\circ} \mathrm{C}$ for 3.5 hours \\
\hline $\begin{array}{l}\text { Maximum operating temperature } \\
\text { requirement }\end{array}$ & $85^{\circ} \mathrm{C}$ \\
\hline
\end{tabular}

Table 2. Summary of the device properties for an ultrathin $9 \AA$ CFB free layer.

corresponding to anti-parallel and parallel states, respectively. Figure $6 \mathrm{~b}$ displays the corresponding resistance versus DC voltage (R-V) loops. R-V measurements consist of sweeping DC voltage between -400 to $400 \mathrm{mV}$. Again, clear switching of the free layer between anti-parallel and parallel states is observed. The change in resistance is consistent between R-H and R-V loops, and corresponds to a TMR of $150 \%$ with a low RA of $3.5 \Omega \cdot \mu \mathrm{m}^{2}$. Note this is not the highest reported TMR for the ultrathin CFB free layer as we have recently reported TMR greater than $180 \%$ for a similar structure with a slightly higher $\mathrm{RA}^{12}$.

Finally, we discuss the high speed switching characteristics of nominal $30 \mathrm{~nm}$ devices summarized in Table 2. Bit error rate tests were first performed with a $10 \mathrm{~ns}$ pulse length at an error rate below $10^{-6}$ or $1 \mathrm{ppm}$. In Fig. $6 \mathrm{c}$, nominal $30 \mathrm{~nm}$, ultrathin CFB free layer devices demonstrate writing at short pulse lengths with a low switching error rate below $1 \mathrm{ppm}$. At $1 \mathrm{ppm}$, the devices exhibit a low writing voltage below $300 \mathrm{mV}$. The low switching voltage is correlated with the thickness of the CFB free layer as shown in Fig. $6 \mathrm{~d}$. While the median $50 \%$ switching voltage V0 shows little dependence on the CFB thickness, V1 clearly shows an increase in writing voltage with free layer thickness which is consistent with the conservation of spin angular momentum ${ }^{12,16}$. As an extension of these findings, we look more closely at the ability of these devices to switch at pulse lengths below $10 \mathrm{~ns}$. Figure $6 \mathrm{e}$ displays the median $50 \%$ switching voltage of nominal $30 \mathrm{~nm}$ diameter devices with $R_{p}$ of $4.7 \mathrm{k} \Omega$ as a function of pulse length ranging from $1 \mathrm{~ns}$ to $50 \mathrm{~ns}$. We observe switching voltages less than $300 \mathrm{mV}$ for pulse lengths of $3 \mathrm{~ns}$ or longer. We also observe switching of the free layer at the fastest switching speed of $1 \mathrm{~ns}$ with a writing voltage below $500 \mathrm{mV}$. These low switching voltages correspond to a low switching energy of $0.056 \mathrm{pJ}$ at $10 \mathrm{~ns}$ and 0.044 $\mathrm{pJ}$ at $1 \mathrm{~ns}$. These findings highlight the importance of a thin, low moment free layer for the design of efficient, low switching voltage MRAM devices, with the inclusion of Mg serving a critical role for enabling thermally stable, ultrathin PMA CFB free layers.

In conclusion, our studies demonstrate the remarkable role of $\mathrm{Mg}$ that enables PMA at ultrathin thicknesses of CoFeB free layers. Without an Mg insertion layer, PMA is first observed for an $11 \AA$ CFB free layer, but with an $\mathrm{Mg}$ insertion layer, PMA is observed in CFB free layers as thin as $6.6 \AA$, the lower limit of this study. A free layer composed of $9 \AA \mathrm{CFB}$ with a nominal $5 \AA \mathrm{Mg}$ insertion layer, followed by a 3.5 hour annealing at $400^{\circ} \mathrm{C}$, exhibits a high $M_{s}$ of $1350 \mathrm{emu} / \mathrm{cm}^{3}$ and $H_{k}$ of $7.3 \mathrm{kOe}$ with anisotropy constants identical to conventional, thicker free layers. For $30 \mathrm{~nm}$ devices, we observe good thermal stability as the calculated upper limit of $\Delta$ is large, approximated at 93 at $25^{\circ} \mathrm{C}$ using a macrospin model, and 85 at $25^{\circ} \mathrm{C}$ using a domain wall model. At device level, a nominal $30 \mathrm{~nm}$ device with a $9 \AA$ free layer exhibits sharp switching of the free layer with a low RA of $3.5 \Omega \cdot \mu \mathrm{m}^{2}$ and TMR 
of $150 \%$. High speed testing reveal these devices are capable of switching at $1 \mathrm{~ns}$ with a writing voltage below $500 \mathrm{mV}$; and below $300 \mathrm{mV}$ for pulse lengths ranging from 3 to $50 \mathrm{~ns}$. Consistent with the conservation of spin angular momentum, an ultrathin free layer is important for the design of efficient, low switching voltage MRAM devices, and thermally stable, ultrathin free layers can be achieved with the incorporation of $\mathrm{Mg}$.

\section{Methods}

All MTJ film stacks presented in this work were prepared using magnetron sputtering in an Anelva C-7100 deposition system. After deposition, the blanket film wafers were annealed at $400^{\circ} \mathrm{C}$ for 3.5 hours. PMA-MTJ films were also patterned into circular devices with diameters of $30 \mathrm{~nm}$ using UV photolithography and etched by a combination of reactive ion etching and argon ion beam etching. Patterned device wafers were annealed at $400^{\circ} \mathrm{C}$ for 3.5 hours only after the completion of the fabrication process. Device diameters are electrical diameters calculated from resistance measurements. VSM was used to measure the out-of-plane magnetic moment for temperatures between $25^{\circ} \mathrm{C}$ and $375^{\circ} \mathrm{C} . M_{s}$ is defined as the magnetic moment normalized by the nominally-deposited ferromagnetic free layer thickness. FMR was used to measure $H_{k}$, with error less than $5 \%$, for temperatures between $30^{\circ} \mathrm{C}$ and $85^{\circ} \mathrm{C}$. Structural characterization was performed using cross-sectional TEM with elemental compositional analysis performed using EDS. TMR is defined as $\frac{R_{A P}-R_{P}}{R_{P}}$. The bit error rate is calculated from data collected from up to $1,200,000$ test cycles, and defined as the number of write errors divided by the number of write errors and successes for a single device.

\section{Data availability}

The datasets generated during and/or analysed during the current study are available from the corresponding author on reasonable request.

Received: 20 December 2018; Accepted: 30 October 2019;

Published online: 19 December 2019

\section{References}

1. Ikeda, S. et al. A perpendicular-anisotropy CoFeB-MgO magnetic tunnel junction. Nat. Mater. 9, 721-724 (2010).

2. Gajek, M. et al. Spin torque switching of $20 \mathrm{~nm}$ magnetic tunnel junctions with perpendicular anisotropy. Appl. Phys. Lett. 100, 132408 (2012).

3. Jan, G. et al. High Spin torque efficiency of magnetic tunnel junctions with $\mathrm{MgO} / \mathrm{CoFeB} / \mathrm{MgO}$ free layer. Appl. Phys. Express 5, 093008 (2012).

4. Kang, S. H. Embedded STT-MRAM for energy-efficient and cost-effective mobile systems. Tech. Dig. Pap. Symp. VLSI Technol. 2014, 5.2 (2014).

5. Slaughter, J. M. et al. High density ST-MRAM technology. Tech. Dig. - Int. Electron Devices Meet. 2012(29), 3 (2012).

6. Jan, G. et al. Achieving Sub-ns switching of STT-MRAM for future embedded LLC applications through improvement of nucleation and propagation switching mechanisms. Tech. Dig. Pap. Symp. VLSI Technol. 2016(2), 4 (2016).

7. Jan, G. et al. Demonstration of fully functional $8 \mathrm{Mb}$ perpendicular STT-MRAM chips with sub-5ns writing for non-volatile embedded memories. Tech. Dig. Pap. Symp. VLSI Technol. 2014, 42 (2014).

8. Lu, Y. et al. Fully Functional perpendicular STT-MRAM macro embedded in $40 \mathrm{~nm}$ logic for energy-efficient IOT applications. Tech. Dig. - Int. Electron Devices Meet. 2015, 26.1 (2015).

9. Shih, M.-C. et al. Reliability study of perpendicular STT-MRAM as emerging embedded memory qualified for reflow soldering at $260^{\circ}$ C. Tech. Dig. Pap. Symp. VLSI Technol. 2016, 14.1 (2016).

10. Koo, J. W. et al. Large perpendicular magnetic anisotropy at Fe/MgO interface. Appl. Phys. Lett. 103, 192401 (2013).

11. Jan, G. et al. Demonstration of Ultra-Low Voltage and Ultra Low Power STT-MRAM designed for compatibility with 0x node embedded LLC applications. Tech. Dig. Pap. Symp. VLSI Technol. 2018, 65 (2018).

12. Thomas, L. et al. STT-MRAM devices with low damping and moment optimized for LLC applications at 0x nodes. Tech. Dig. Int. Electron Devices Meet. 2018, 27.3 (2018).

13. Liu, T., Zhang, Y. \& Pan, H. Y. Thermally robust $\mathrm{Mo} / \mathrm{CoFeB} / \mathrm{MgO} /$ trilayers with strong perpendicular magnetic anisotropy. Sci. Rep. 4, 5895 (2014).

14. Kim, J.-H. et al. Ultrathin W space layer-enabled thermal stability enhancement in a perpendicular $\mathrm{MgO} / \mathrm{CoFeB} / \mathrm{W} / \mathrm{CoFeB} / \mathrm{MgO}$ recording frame. Sci. Rep. 5, 16903 (2015).

15. Iwata-Harms, J. M. et al. High-temperature thermal stability driven by magnetization dilution in CoFeB free layers for spin-transfertorque magnetic random access memory. Sci. Rep. 8, 14409 (2018).

16. Slonczewski, J. C. Current-driven excitation of magnetic multilayers. J. Magn. Magn. Mater. 159, L1 (1996).

17. Callen, E. \& Callen, H. Ferromagnetic transitions and the one-third-power law. J. Appl. Phys. 36, 1140 (1965).

18. Kuz'min, M. D. Shape of temperature dependence of spontaneous magnetization of ferromagnets: quantitative analysis. Phys. Rev. Lett. 94, 107204 (2005).

19. Stoner, E. C. \& Wohlfarth, E. P. A mechanism of magnetic hysteresis in heterogeneous alloys. Phil. Trans. R. Soc. Lond. A 240, 599-642 (1948).

20. Thomas, L. et al. Solving the paradox of the inconsistent size dependence of thermal stability at device and chip-level in perpendicular STT-MRAM. Tech. Dig. Int. Electron Devices Meet. 2015, 26.4 (2015).

21. Herring, C. \& Kittel, C. On the theory of spin waves in ferromagnetic media. Phys. Rev. 81, 869 (1951).

22. Luo, Y.-R. Bond disassociation energies in Comprehensive Handbook of Chemical Bond Energies (ed. Lide, D. R.) 574, 803,855 (CRC Press, 2007).

\section{Author contributions}

P.-K.W. supervised the study. J.M.I.-H. and G.J. designed the study. J.M.I.-H. and G.J. wrote the manuscript. J.M.I.-H., S.S.G., L.T., H.L., J.Z. and Y.-J.L. performed measurements, data analysis, and data interpretation. R.T., S.P. and V.S. performed the thin film deposition for all samples used in this study. D.S. and Y.Y. developed the etching processes for all device samples used in this study. R.H., J.H., Z.T., V.L. and P.L. developed and oversaw the integration of MTJ thin films into devices. Y.-J.W., T.Z. and H.F. supervised the processing of all blanket film and patterned device wafers used in this study. S.L. provided support for the testing and characterization equipment. D.S. provided assistance with TEM studies. All authors discussed the results and reviewed the manuscript. 


\section{Competing interests}

The authors declare no competing interests.

\section{Additional information}

Correspondence and requests for materials should be addressed to J.M.I.-H.

Reprints and permissions information is available at www.nature.com/reprints.

Publisher's note Springer Nature remains neutral with regard to jurisdictional claims in published maps and institutional affiliations.

(c) (i) Open Access This article is licensed under a Creative Commons Attribution 4.0 International License, which permits use, sharing, adaptation, distribution and reproduction in any medium or format, as long as you give appropriate credit to the original author(s) and the source, provide a link to the Creative Commons license, and indicate if changes were made. The images or other third party material in this article are included in the article's Creative Commons license, unless indicated otherwise in a credit line to the material. If material is not included in the article's Creative Commons license and your intended use is not permitted by statutory regulation or exceeds the permitted use, you will need to obtain permission directly from the copyright holder. To view a copy of this license, visit http://creativecommons.org/licenses/by/4.0/.

(c) The Author(s) 2019 